\title{
The Application of Vector Lyapunov Functions in Iterative Learning Control
}

\author{
Qunli Zhang* \\ College of Mathematics and Statistics, Heze University, Heze, Shandong 274015, China \\ *Corresponding author
}

\begin{abstract}
This paper presents iterative learning control of multiple state vector system, whose initial states are equal. By introducing the vector Lyapunov function, it involves interesting criteria to guarantee the robust convergence of the tracking error in the sense of the $\lambda$ - norm. Finally, the validity of the proposed method is verified by an example.
\end{abstract}

Keywords-iterative learning control; convergence; vector lyapunov function; $\lambda-$ norm

\section{INTRODUCTION}

Since iterative learning control, which belongs to the intelligent control methodology, is proposed by Arimoto et al. in 1984(See[1]), this feed-forward control approach for fully utilizing the previous control information and improving the transient performance of studied systems that is suitable for repetitive movements has been a major research area and a hot issue in recent years. Its goal is to get full range of tracking tasks on finite interval (See [2-15]).

It is well known that the more complex the considered dynamical system is, the more difficult it is to find a Lyapunov function. This arouse us to employ several Lyapunov function, which is called to be a vector Lyapunov function, for each component provides information about a part of the dynamics. Hence, the corresponding theory, namely, the method of vector Lyapunov function, offers a very flexible process (see [16-22]).

There are few research results on iterative learning control for systems with time-varying coefficient as well as vector Lyapunov function. In this paper, using vector Lyapunov function and $\lambda-$ norm, we obtain some sufficient conditions to guarantee the output states of the considered time-varying coefficient system to converge to the desired trajectories. It is shown that the proposed method can achieve the ILC convergence property significantly.

Before ending this section, it is worth pointing out the main contribution of this paper is the design of iterative controllers from vector Lyapunov function, which is different from the designed controllers by positive definite Lyapunov function, in past literature.

\section{PRELIMINARIES}

Throughout this paper, the 2-norm for the $n$ - dimensional vector $\quad x=\left(x_{1}, x_{2}, \cdots, x_{n}\right)^{T}$ is defined as $\|x\|=\left(\sum_{i=1}^{n} x_{i}\right)^{1 / 2}$ , while the $\lambda-$ norm for a function is defined $\|\cdot\|_{\lambda}=\sup _{t \in[0, T]}\left\{e^{-\lambda t}\|\cdot\|\right\}$, where the superscript $T$ represents the transpose and $\lambda>0 .|A|=\left(\left|a_{i j}\right|\right)_{n \times n}$, where $A=\left(a_{i j}\right)_{n \times n} \in R^{n \times n}$ is a matrix.

$$
\begin{gathered}
\text { Lemma } \\
\sup _{t \in[0, T]}\left\{e^{-\lambda t} \int_{0}^{t}\|x(\tau)\| d \tau\right\} \leq \frac{1}{\lambda}\|x(t)\|_{\lambda} .
\end{gathered}
$$

Consider

\section{MAIN RESULTS}

Consider the following multiple state vector system

$$
\begin{gathered}
\dot{x}_{k}=F\left(t, x_{k}, y_{k}\right)+u_{x, k}(t), \\
z_{x, k}(t)=C x_{k}+D u_{x, k}(t), \\
u_{x, k+1}(t)=u_{x, k}(t)+M e_{x, k}(t), \\
\dot{y}_{k}=G\left(t, x_{k}, y_{k}\right)+u_{y, k}(t), \\
z_{y, k}(t)=C y_{k}+D u_{y, k}(t), \\
u_{y, k+1}(t)=u_{y, k}(t)+M e_{y, k}(t),
\end{gathered}
$$

where $\quad x_{k}, y_{k} \in R^{n}$ are the state vectors, $u_{x, k}, u_{y, k} \in R^{n}$ are input vectors of $x_{k}, y_{k}$, and $z_{x, k}, z_{y, k} \in R^{n}$ are output vectors of $x_{k}, y_{k}$, respectively. $k$ is the number of iterations, $k \in\{1,2,3, \cdots\}$ and $t \in[0, T], T$ is a constant.

Let 
$e_{x, k}(t)=z_{x, d}(t)-z_{x, k}(t), e_{y, k}(t)=z_{y, d}(t)-z_{y, k}(t)$, where $z_{x, d}(t), z_{y, d}(t)$ are reference outputs of $x_{k}, y_{k}$, respectively. So we have

$$
\begin{aligned}
e_{x, k+1}(t)= & z_{x, d}(t)-z_{x, k+1}(t)=z_{x, d}(t)-z_{x, k}(t)+z_{x, k}(t)-z_{x, k+1}(t) \\
= & e_{x, k}(t)+z_{x, k}(t)-z_{x, k+1}(t) \\
& e_{y, k+1}(t)=e_{y, k}(t)+z_{y, k}(t)-z_{y, k+1}(t)
\end{aligned}
$$

We define the operator $V: R^{n} \times R^{n} \rightarrow R^{n}$ such that

(1) $V(0,0)=0$;

(2) $V(x+z, y+w)=V(x, y)+V(z, w)$ for any vectors $x, z, y, w \in R^{n}$,

(3) there is a constant $\gamma>0$ such that $V(C x, C y)=C^{\gamma} V(x, y)$ for any vectors $x, y \in R^{n}$ and matrix $C \in R^{n \times n}$;

(4) the derivative $\frac{\partial V}{\partial x}$ and $\frac{\partial V}{\partial y}$ of $V(x, y)$ exist, where $x, y \in R^{n}$

For the sake of convenient, we denote that the set, whose elements are operators $V: R^{n} \times R^{n} \rightarrow R^{n}$ and satisfy the above four conditions, is $\aleph$.

For any operator $V \in \aleph$, the following conclusion can be gotten

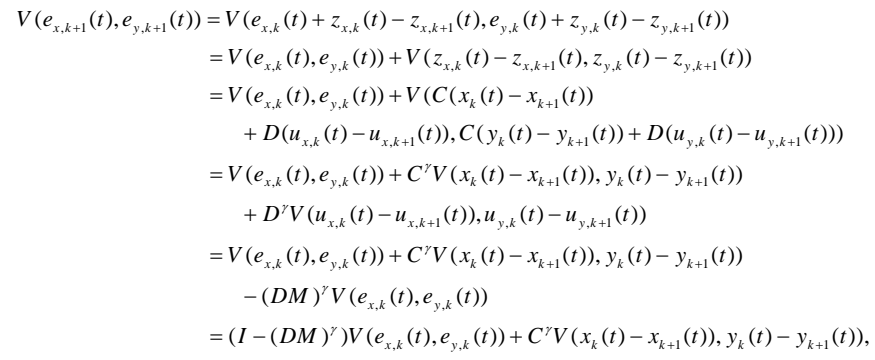

where $I$ is identical matrix. In fact:

$$
\begin{aligned}
& z_{x, k}(t)-z_{x, k+1}(t)=C\left(x_{k}(t)-x_{k+1}(t)\right)+D\left(u_{x, k}(t)-u_{x, k+1}(t)\right), \\
& z_{y, k}(t)-z_{y, k+1}(t)=C\left(y_{k}(t)-y_{k+1}(t)\right)+D\left(u_{y, k}(t)-u_{y, k+1}(t)\right),
\end{aligned}
$$

$u_{x, k}(t)-u_{x, k+1}(t)=-M e_{x, k}(t), u_{y, k}(t)-u_{y, k+1}(t)=-M e_{y, k}(t)$.

Then from (3) we can obtain that

$\left.\left\|V\left(e_{x, k+1}(t), e_{y, k+1}(t)\right)\right\| \leq\left\|I-(D M)^{y}\right\| \cdot\left\|V\left(e_{x, k}(t), e_{y, k}(t)\right)\right\|+\left\|C^{n}\right\| \| V\left(x_{k}(t)-x_{k+1}(t)\right), y_{k}(t)-y_{k+1}(t)\right) \|$.

$$
\left.\underset{\text { have }}{\text { For }} \| V\left(x_{k}(t)-x_{k+1}(t)\right), y_{k}(t)-y_{k+1}(t)\right) \| \text { in (4), we }
$$

$$
\begin{aligned}
& \frac{d e^{r t} V\left(x_{k}(t)-x_{k+1}(t), y_{k}(t)-y_{k+1}(t)\right)}{d t}=r e^{r t} V\left(x_{k}(t)-x_{k+1}(t), y_{k}(t)-y_{k+1}(t)\right) \\
&+e^{r t}\left(\frac{\partial V}{\partial\left(x_{k}(t)-x_{k+1}(t)\right)} \cdot \frac{d\left(x_{k}(t)-x_{k+1}(t)\right)}{d t}\right) \\
&+e^{r t}\left(\frac{\partial V}{\partial\left(y_{k}(t)-y_{k+1}(t)\right)} \cdot \frac{d\left(y_{k}(t)-y_{k+1}(t)\right)}{d t}\right) \\
&=r e^{r t} V\left(x_{k}(t)-x_{k+1}(t), y_{k}(t)-y_{k+1}(t)\right) \\
&+e^{r t}\left(\frac{\partial V}{\partial\left(x_{k}(t)-x_{k+1}(t)\right)} \cdot\left(F\left(t, x_{k}, y_{k}\right)-F\left(t, x_{k+1}, y_{k+1}\right)\right)\right) \\
&+e^{r t}\left(\frac{\partial V}{\partial\left(y_{k}(t)-y_{k+1}(t)\right)} \cdot\left(G\left(t, x_{k}, y_{k}\right)-G\left(t, x_{k+1}, y_{k+1}\right)\right)\right)
\end{aligned}
$$

$$
\begin{aligned}
& +e^{r t}\left(\frac{\partial V}{\partial\left(x_{k}(t)-x_{k+1}(t)\right)} \cdot\left(-M e_{x, k}\right)\right) \\
& +e^{r t}\left(\frac{\partial V}{\partial\left(y_{k}(t)-y_{k+1}(t)\right)} \cdot\left(-M e_{y, k}\right)\right)
\end{aligned}
$$

$$
\begin{aligned}
= & r e^{r t} V_{k}(t)+e^{r t}\left(V_{x, k}(t) \cdot f_{k}(t)+V_{y, k}(t) \cdot g_{k}(t)\right) \\
& -e^{r t}\left(V_{x, k}(t) \cdot M e_{x, k}+V_{y, k}(t) \cdot M e_{y, k}\right) .
\end{aligned}
$$

$$
\begin{aligned}
& \text { where } V\left(x_{k}(t)-x_{k+1}(t), y_{k}(t)-y_{k+1}(t)\right)=V_{k}(t) \\
& \frac{\partial V_{k}}{\partial\left(x_{k}(t)-x_{k+1}(t)\right)}=V_{x, k}, \frac{\partial V_{k}}{\partial\left(y_{k}(t)-y_{k+1}(t)\right)}=V_{y, k} \\
& F\left(t, x_{k}, y_{k}\right)-F\left(t, x_{k+1}, y_{k+1}\right)=f_{k}(t), \\
& G\left(t, x_{k}, y_{k}\right)-F\left(t, x_{k+1}, y_{k+1}\right)=g_{k}(t) .
\end{aligned}
$$

we integrate $e^{r t} V_{k}(t)$ with respect to $t$ and obtain

$e^{r t} V_{k}(t)=e^{r s} V_{k}(s)+\int_{s}^{t}\left[r e^{r \rho} V_{k}(\rho)+e^{r u}\left(V_{x, k}(\rho) \cdot f_{k}(\rho)+V_{y, k}(\rho) \cdot g_{k}(\rho)\right)\right] d \rho$ $-\int_{s}^{t} e^{r \rho}\left(V_{x, k}(\rho) \cdot M e_{x, k}+V_{y, k}(\rho) \cdot M e_{y, k}\right) d \rho$ 
$e^{r t}\left\|V_{k}(t)\right\|=e^{r s}\left\|V_{k}(s)\right\|+\int_{s}^{t} e^{r \rho}\left\|r V_{k}(\rho)+\left(V_{x, k}(\rho) \cdot f_{k}(\rho)+V_{y, k}(\rho) \cdot g_{k}(\rho)\right)\right\| d \rho$

$$
+\int_{s}^{t} e^{r \rho}\left\|V_{x, k}(\rho) \cdot M e_{x, k}+V_{y, k}(\rho) \cdot M e_{y, k}\right\| d \rho
$$

$r e^{r t}\left\|V_{k}(t)\right\|+e^{r t}\left\|V_{k}(t)\right\|_{t}^{\prime}=e^{r t}\left\|r V_{k}(t)+\left(V_{x, k}(t) \cdot f_{k}(t)+V_{y, k}(t) \cdot g_{k}(t)\right)\right\|$ $+e^{r t}\left\|V_{x, k}(t) \cdot M e_{x, k}+V_{y, k}(t) \cdot M e_{y, k}\right\|$.

If there exists a constant $\varpi$ such that

$$
\left\|V_{x, k}(t) \cdot M e_{x, k}+V_{y, k}(t) \cdot M e_{y, k}\right\| \leq \varpi\left\|V\left(e_{x, k}(t), e_{y, k}(t)\right)\right\|,
$$

the following conclusion

$$
\begin{gathered}
\left\|V_{k}(t)\right\|_{t}^{\prime} \leq\left\|r V_{k}(t)+\left(V_{x, k}(t) \cdot f_{k}(t)+V_{y, k}(t) \cdot g_{k}(t)\right)\right\|-r\left\|V_{k}(t)\right\| \\
+\varpi\left\|V\left(e_{x, k}(t), e_{y, k}(t)\right)\right\|
\end{gathered}
$$

is true.

$\begin{array}{clr}\text { It is easy to prove that } \\ \left\|r V_{k}(t)+\left(V_{x, k}(t) \cdot f_{k}(t)+V_{y, k}(t) \cdot g_{k}(t)\right)\right\|-r\left\|V_{k}(t)\right\| & \text { is }\end{array}$

monotonically

decreasing on $r$, thus the limit $\lim _{r \rightarrow+\infty}\left(\left\|r V_{k}(t)+\left(V_{x, k}(t) \cdot f_{k}(t)+V_{y, k}(t) \cdot g_{k}(t)\right)\right\|-r\left\|V_{k}(t)\right\|\right) \quad$ exists. From 2-norm of the $n$ - dimensional vector, we obtain

$$
\begin{gathered}
\lim _{r \rightarrow+\infty}\left(\left\|r V_{k}(t)+\left(V_{x, k}(t) \cdot f_{k}(t)+V_{y, k}(t) \cdot g_{k}(t)\right)\right\|-r\left\|V_{k}(t)\right\|\right) \\
=\frac{\left(V_{x, k}(t) \cdot f_{k}(t)+V_{y, k}(t) \cdot g_{k}(t)\right)^{T} V_{k}(t)}{\left\|V_{k}(t)\right\|}=h_{k}(t) .
\end{gathered}
$$

$$
\left\|V_{k}(t)\right\|_{t}^{\prime} \leq h_{k}(t)+\varpi\left\|V\left(e_{x, k}(t), e_{y, k}(t)\right)\right\|
$$

Based on the above calculation, the following theorem is obtained.

Theorem 1 If the system (1) and the operator $V \in \aleph$ satisfy the condition (5), then the conclusions (4) and (7) are true.

$$
\begin{aligned}
& \text { Corollary } 1 \text { when } x_{k}(t) \in R^{n}, y_{k}(t) \in R^{m} \text {, and } \\
& n>m \text {, let } y_{k}(t) \rightarrow\left(\begin{array}{c}
y_{k}(t) \\
0_{n-m}
\end{array}\right)=\tilde{y}_{k}(t), \\
& G\left(t, x_{k}(t), y_{k}(t)\right) \rightarrow\left(\begin{array}{c}
G\left(t, x_{k}(t), y_{k}(t)\right) \\
0_{n-m}
\end{array}\right)=\tilde{G}\left(t, x_{k}(t), \tilde{y}_{k}(t)\right),
\end{aligned}
$$

one obtains the similar result with above Theorem.

Remark When there are three parts $x_{k}, y_{k}, z_{k}$ about the multiple state vector system (1), we can construct $V\left(e_{x, k}(t), e_{y, k}(t), e_{z, k}(t)\right) \in \aleph$ the similar conclusion with Theorem 1 . $\begin{array}{cccc}\text { Corollary } & 2 & \text { For } & \text { system } \\ \text { (1), }\end{array}$

taking

$V\left(x_{k}(t)-x_{k+1}(t), y_{k}(t)-y_{k+1}(t)\right)$

$=\alpha\left(x_{k}(t)-x_{k+1}(t)\right)+\beta\left(y_{k}(t)-y_{k+1}(t)\right), \quad$ the following results are drawn:

$\left\|\alpha e_{x, k+1}(t)+\beta e_{y, k+1}(t)\right\| \leq\|I-D M\|\left\|\alpha e_{x, k}(t)+\beta e_{y, k}(t)\right\|+\|C\|\left\|\alpha\left(x_{k}-x_{k+1}\right)+\beta\left(y_{k}-y_{k+1}\right)\right\|$,

$$
\left\|p_{k}(t)\right\|_{t}^{\prime} \leq \frac{p_{k}^{T}(t) q_{k}(t)}{\left\|p_{k}(t)\right\|}+\|M\|\left\|\alpha e_{x, k}(t)+\beta e_{y, k}(t)\right\|
$$

where

$$
\begin{aligned}
& p_{k}(t)=\alpha\left(x_{k}-x_{k+1}\right)+\beta\left(y_{k}-y_{k+1}\right), \\
& q_{k}(t)=\alpha\left[F\left(t, x_{k}, y_{k}\right)-F\left(t, x_{k+1}, y_{k+1}\right)\right]
\end{aligned}
$$

$+\beta\left[G\left(t, x_{k}, y_{k}\right)-G\left(t, x_{k+1}, y_{k+1}\right)\right]$.

In fact,

$$
\begin{aligned}
\alpha e_{x, k+1}(t)+\beta e_{y, k+1}(t)= & \alpha e_{x, k}(t)+\beta e_{y, k}(t)+\alpha\left(z_{x, k}-z_{x, k+1}\right) \\
& +\beta\left(z_{y, k}-z_{y, k+1}\right) \\
= & \left(\alpha e_{x, k}(t)+\beta e_{y, k}(t)\right)+\alpha\left[C\left(x_{k}-x_{k+1}\right)+D\left(u_{x, k}-u_{x, k+1}\right)\right] \\
& +\beta\left[C\left(y_{k}-y_{k+1}\right)+D\left(u_{y, k}-u_{y, k+1}\right)\right] \\
= & (I-D M)\left(\alpha e_{x, k}(t)+\beta e_{y, k}(t)\right) \\
& +C\left[\alpha\left(x_{k}-x_{k+1}\right)+\beta\left(y_{k}-y_{k+1}\right)\right] .
\end{aligned}
$$

Imitating the inference of (7), we have

$$
\frac{d e^{r t} p_{k}(t)}{d t}=r e^{r t} p_{k}(t)+e^{r t} q_{k}(t)-e^{r t} M\left[\alpha e_{x, k}(t)+\beta e_{y, k}(t)\right]
$$

$e^{r t} p_{k}(t)=e^{r s} p_{k}(s)+\int_{s}^{t} e^{r u}\left[r p_{k}(u)+q_{k}(u)-M\left(\alpha e_{x, k}(u)+\beta e_{y, k}(u)\right)\right] d u$,

$e^{r t}\left\|p_{k}(t)\right\| \leq e^{r s}\left\|p_{k}(s)\right\|+\int_{s}^{t} e^{r u}\left\|r p_{k}(u)+q_{k}(u)\right\| d u+\int_{s}^{t}\|M\|\left\|\alpha e_{x, k}(u)+\beta e_{y, k}(u)\right\| d u$,

$e^{r t}\left\|p_{k}(t)\right\|_{t}^{\prime} \leq e^{r t}\left[\left\|r p_{k}(t)+q_{k}(t)\right\|+\|M\|\left\|\alpha e_{x, k}(t)+\beta e_{y, k}(t)\right\|\right]-r e^{r t}\left\|p_{k}(t)\right\|$.

Going on to the next item, we will infer the important 
conclusion of this paper.

Conditions (A), (B) are satisfied if the following conditions hold:

(A) The operator $V_{k, i}(t) \in \aleph, i=1,2$.There exist functions $p_{j i}(t), i, j=1,2$, and such that

$$
\frac{\left(V_{x, k, i}(t) \cdot f_{k}(t)+V_{y, k, i}(t) \cdot g_{k}(t)\right)^{T} V_{k, i}(t)}{\left\|V_{k, i}(t)\right\| \cdot\left\|V_{k, j}(t)\right\|} \leq p_{j i}(t)
$$

$\left\|V_{x, k, i}(t) \cdot M e_{x, k}+V_{y, k, i}(t) \cdot M e_{y, k}\right\| \leq \varpi_{i 1}\left\|V_{1}\left(e_{x, k}(t), e_{y, k}(t)\right)\right\|+\varpi_{i 2}\left\|V_{2}\left(e_{x, k}(t), e_{y, k}(t)\right)\right\|$, where $\varpi_{i j}$ are constants.

Theorem 2 Suppose the operator $F, G$ in system (1) satisfy $F(t, 0,0)=G(t, 0,0)=0$. There are operators $V_{k, i}(t) \in \aleph, V_{i}\left(e_{x, k}(t), e_{y, k}(t)\right), i=1,2$, such that conditions (A),(B), and

$$
V_{i}\left(e_{x, k}(t), e_{y, k}(t)\right)=0 \text { if and only if } e_{x, k}(t)=e_{y, k}(t)=0
$$

If there exists a constant $\lambda>0$ such that initial state vector $x_{k}(0)-x_{k+1}(0)=0, y_{k}(0)-y_{k+1}(0)=0$, and

$$
\lim _{k \rightarrow+\infty} Q^{k}(t)=\left(\begin{array}{ll}
0 & 0 \\
0 & 0
\end{array}\right)
$$

\section{where}

$Q(t)=\left(\begin{array}{cc}\left\|I-(D M)^{\gamma}\right\| & 0 \\ 0 & \left\|I-(D M)^{\gamma}\right\|\end{array}\right)+\left(\begin{array}{cc}\left\|C^{\gamma}\right\| & 0 \\ 0 & \left\|C^{\gamma}\right\|\end{array}\right) \cdot|\Phi(t)| \cdot \frac{B(t)}{\lambda}, \quad \Phi(t)$ is a basic solution matrix of the system $\left(\begin{array}{l}\left\|V_{k, 1}(t)\right\| \\ \left\|V_{k, 2}(t)\right\|^{\prime}\end{array}\right)_{t}^{\prime} \leq\left(\begin{array}{ll}p_{11}(t) & p_{12}(t) \\ p_{21}(t) & p_{22}(t)\end{array}\right)\left(\begin{array}{l}\left\|V_{k, 1}(t)\right\| \\ \left\|V_{k, 2}(t)\right\|\end{array}\right), B(t)=\mid \Phi^{-1}(t)\left(\begin{array}{ll}\varpi_{11} & \varpi_{12} \\ \varpi_{21} & \varpi_{22}\end{array}\right)$, then the system (1) can guarantee that $z_{x, k}(t), z_{y, k}(t)$ can track $z_{x, d}(t), z_{y, d}(t)$, respectively.

Proof From conditions (A), (B) and the above inference, we can obtain

$$
\left(\begin{array}{l}
\left\|V_{k, 1}(t)\right\| \\
\left\|V_{k, 2}(t)\right\|
\end{array}\right)_{t}^{\prime} \leq\left(\begin{array}{ll}
p_{11}(t) & p_{12}(t) \\
p_{21}(t) & p_{22}(t)
\end{array}\right)\left(\begin{array}{l}
\left\|V_{k, 1}(t)\right\| \\
\left\|V_{k, 2}(t)\right\|
\end{array}\right)+\left(\begin{array}{cc}
\varpi_{11} & \varpi_{12} \\
\varpi_{21} & \varpi_{22}
\end{array}\right)\left(\begin{array}{l}
\left\|V_{1}\left(e_{x, k}(t), e_{y, k}(t)\right)\right\| \\
\left\|V_{2}\left(e_{x, k}(t), e_{y, k}(t)\right)\right\|
\end{array}\right) .
$$

From (1)-(7) and (12), the following conclusion

$$
\left(\begin{array}{l}
\left\|V_{k, 1}(t)\right\| \\
\left\|V_{k, 2}(t)\right\|
\end{array}\right) \leq \Phi(t) \cdot \int_{0}^{t} \Phi^{-1}(\rho)\left(\begin{array}{ll}
\varpi_{11} & \varpi_{12} \\
\varpi_{21} & \varpi_{22}
\end{array}\right)\left(\begin{array}{l}
\left\|V_{1}\left(e_{x, k}(\rho), e_{y, k}(\rho)\right)\right\| \\
\left\|V_{2}\left(e_{x, k}(\rho), e_{y, k}(\rho)\right)\right\|
\end{array}\right) d \rho
$$

$$
\begin{aligned}
& \text { is true because } x_{k}(0)-x_{k+1}(0)=0 \text {, } \\
& y_{k}(0)-y_{k+1}(0)=0 \text {. } \\
& +\left(\begin{array}{cc}
\left\|C^{r}\right\| & 0 \\
0 & \left\|C^{\gamma}\right\|
\end{array}\right) \cdot \Phi(t) \cdot \int_{0}^{t} \Phi^{-1}(\rho)\left(\begin{array}{ll}
\varpi_{11} & \varpi_{12} \\
\varpi_{21} & \varpi_{22}
\end{array}\right)\left(\begin{array}{l}
\left\|V_{1}\left(e_{x, k}(\rho), e_{y, k}(\rho)\right)\right\| \\
\left\|V_{2}\left(e_{x, k}(\rho), e_{y, k}(\rho)\right)\right\|
\end{array}\right) d \rho .
\end{aligned}
$$

Taking $\lambda$-norm, we have

$$
\begin{aligned}
& \left(\begin{array}{l}
\left\|V_{1}\left(e_{x, k+1}(t), e_{y, k+1}(t)\right)\right\|_{\lambda} \\
\left\|V_{2}\left(e_{x, k+1}(t), e_{y, k+1}(t)\right)\right\|_{\lambda}
\end{array}\right) \leq\left(\begin{array}{cc}
\left\|E-(D M)^{\gamma}\right\| & 0 \\
0 & \left\|E-(D M)^{\gamma}\right\|
\end{array}\right)\left(\begin{array}{l}
\left\|V_{1}\left(e_{x, k}(t), e_{y, k}(t)\right)\right\|_{\lambda} \\
\left\|V_{2}\left(e_{x, k}(t), e_{y, k}(t)\right)\right\|_{\lambda}
\end{array}\right) \\
& +\left(\begin{array}{cc}
\left\|C^{\gamma}\right\| & 0 \\
0 & \left\|C^{\gamma}\right\|
\end{array}\right) \cdot|\Phi(t)| \cdot \frac{B(t)}{\lambda}\left(\begin{array}{l}
\left\|V_{1}\left(e_{x, k}(t), e_{y, k}(t)\right)\right\|_{\lambda} \\
\left\|V_{2}\left(e_{x, k}(t), e_{y, k}(t)\right)\right\|_{\lambda}
\end{array}\right) \\
& =Q(t) \cdot\left(\begin{array}{l}
\left\|V_{1}\left(e_{x, k}(t), e_{y, k}(t)\right)\right\|_{\lambda} \\
\left\|V_{2}\left(e_{x, k}(t), e_{y, k}(t)\right)\right\|_{\lambda}
\end{array}\right) \text {. }
\end{aligned}
$$

when the condition (11) holds, we have $\lim _{k \rightarrow+\infty}\left(\begin{array}{l}\left\|V_{1}\left(e_{x, k}(t), e_{y, k}(t)\right)\right\|_{\lambda} \\ \left\|V_{2}\left(e_{x, k}(t), e_{y, k}(t)\right)\right\|_{\lambda}\end{array}\right)=\left(\begin{array}{l}0 \\ 0\end{array}\right) . \quad$ That implies $\lim _{k \rightarrow+\infty}\left\|V_{j}\left(e_{x, k}(t), e_{y, k}(t)\right)\right\|_{\lambda}=0, j=1,2 \lim _{k \rightarrow+\infty}\left\|V_{j}\left(e_{x, k}(t), e_{y, k}(t)\right)\right\|=0, \quad$ i.e. $\lim _{k \rightarrow+\infty} e_{x, k}(t)=0, \lim _{k \rightarrow+\infty} e_{y, k}(t)=0$ from the condition (10).

Corollary 3 Suppose the operator $F, G$ in system (1) satisfy $F(t, 0,0)=G(t, 0,0)=0$. There are operators $V_{i}\left(e_{x, k}(t), e_{y, k}(t)\right)=\alpha_{i} e_{x, k}(t)+\beta_{i} e_{y, k}(t), i=1,2$, such that conditions (A), and

$\alpha_{i} e_{x, k}(t)+\beta_{i} e_{y, k}(t)=0, i=1,2$, if and only if $e_{x, k}(t)=e_{y, k}(t)=0$.

If there exists a constant $\lambda>0$ such that initial state vector $x_{k}(t)-x_{k+1}(t)=0, y_{k}(t)-y_{k+1}(t)=0$, and

$$
\lim _{k \rightarrow+\infty} Q^{k}(t)=\left(\begin{array}{ll}
0 & 0 \\
0 & 0
\end{array}\right)
$$

where

$Q(t)=\left(\begin{array}{cc}\left\|I-(D M)^{\gamma}\right\| & 0 \\ 0 & \left\|I-(D M)^{\gamma}\right\|\end{array}\right)+\left(\begin{array}{cc}\left\|C^{\gamma}\right\| & 0 \\ 0 & \left\|C^{\gamma}\right\|\end{array}\right) \cdot|\Phi(t)| \cdot \frac{B(t)}{\lambda}$, 
$\Phi(t)$ is a basic solution matrix of the system $\left(\begin{array}{l}\left\|V_{k, 1}(t)\right\| \\ \left\|V_{k, 2}(t)\right\|\end{array}\right)_{t}^{\prime} \leq\left(\begin{array}{ll}p_{11}(t) & p_{12}(t) \\ p_{21}(t) & p_{22}(t)\end{array}\right)\left(\begin{array}{l}\left\|V_{k, 1}(t)\right\| \\ \left\|V_{k, 2}(t)\right\|\end{array}\right)$, $B(t)=\left|\Phi^{-1}(t)\right|$, then the system (1) can guarantee that $z_{x, k}(t), z_{y, k}(t)$ can track $z_{x, d}(t), z_{y, d}(t)$, respectively.

\section{EXAMPLE}

Considering the system

$$
\begin{aligned}
& \dot{x}_{k}=0.2 e^{-t} x_{k}+0.3 y_{k} \sin t+u_{x, k}, \\
& z_{x, k}=0.03 x_{k}+0.3 u_{x, k}, \\
& u_{x, k+1}=u_{x, k}+3 e_{x, k}, \\
& \dot{y}_{k}=0.3 x_{k} \sin t+0.2 e^{-t} y_{k}+u_{y, k}, \\
& z_{y, k}=0.03 y_{k}+0.3 u_{y, k}, \\
& u_{y, k+1}=u_{y, k}+3 e_{y, k} .
\end{aligned}
$$

We take $T=3$, that is $t \in[0,3]$, and $z_{x, d}(t)=\sin t, z_{y, d}(t)=\cos t$. It is easy to verify this example satisfies the conditions of Theorem 2 when $V_{1}\left(e_{x, k}(t), e_{y, k}(t)\right)=\left(e_{x, k}(t)+e_{y, k}(t)\right)^{2}$,

$$
V_{2}\left(e_{x, k}(t), e_{y, k}(t)\right)=\left(e_{x, k}(t)-e_{y, k}(t)\right)^{2} \text {, and satisfies the }
$$
conditions of Corollary 3 when $V_{1}\left(e_{x, k}(t), e_{y, k}(t)\right)$

$$
=\alpha e_{x, k}(t)+\beta e_{y, k}(t), V_{2}\left(e_{x, k}(t), e_{y, k}(t)\right)
$$

$=\beta e_{x, k}(t)+\alpha e_{y, k}(t)$. In following Figure I and Figure II, the output errors $e_{x, k}(t), e_{y, k}(t)$ are exhibited at iteration $k=4, k=5$, respectively.

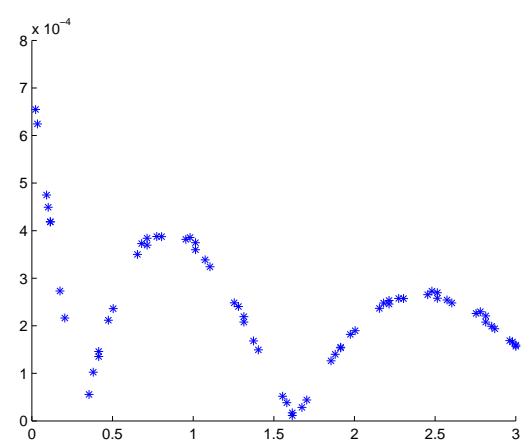

FIGURE I. ERROR $e_{x, k}(t)$ AFTER ITERATION 4

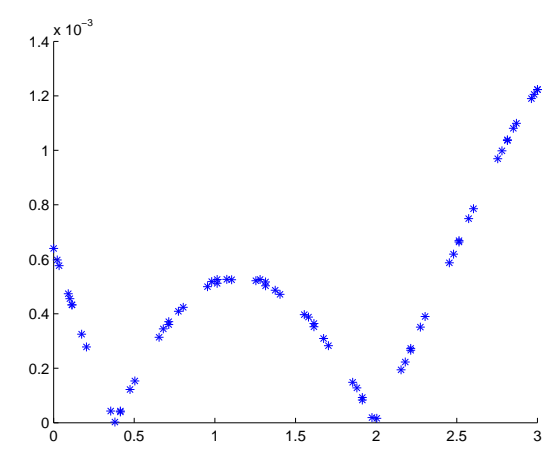

FIGURE II. ERROR $e_{y, k}(t)$ AFTER ITERATION 4

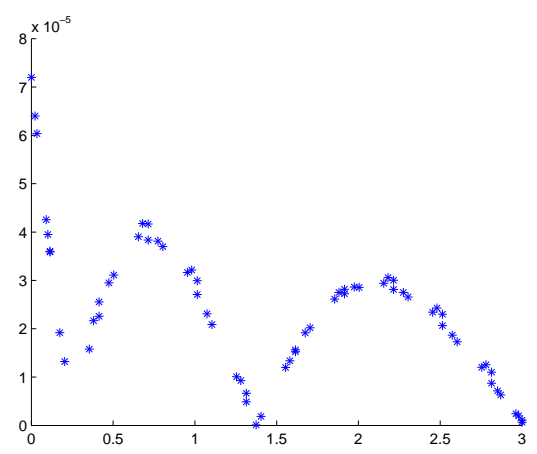

FIGURE III. ERROR $e_{x, k}(t)$ AFTER ITERATION 5

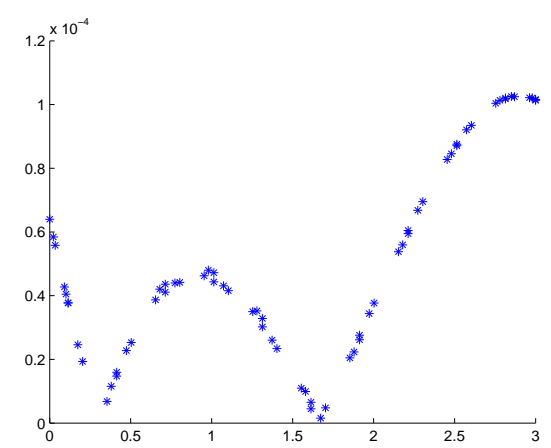

FIGURE IV. ERROR $e_{y, k}(t)$ AFTER ITERATION 5

\section{CONCLUSION}

In this paper, considering the iterative learning control problem for a class of systems, and combining with vector Lyapunov function, the novel controllers, which can guarantee the robust convergence of the tracking error, are designed. From Figs.1and 2 of the given example, we find that the output errors $e_{x, k}(t), e_{y, k}(t)$ downsize almost 10 times from iteration 4 to iteration 5. So it is made known that the proposed method is effective. 


\section{ACKNOWLEDGMENTS}

This work is supported by the National Natural Science Foundation of China (11371221), the Natural Science Foundation of Shandong Province of China (ZR2014AM032), and the Project of Shandong Province Higher Educational Science and Technology Program (J16LI15).

\section{REFERENCE}

[1] S. Arimoto, S. Kawamura, and F. Miyazaki. Bettering operation of robots by learning, Journal of Robotic Systems, 1(2), pp.123-140, 1984.

[2] H.-S. Lee and Z. Bien, A note on convergence property of iterative learning controller with respect to sup norm, Automatica, vol.33, no.8, pp.1591-1593, 1997.

[3] Y. Chen, Z. Gong, and C. Wen. Analysis of a high-order iterative learning control algorithm for uncertain nonlinear systems with state delays, Automatica, vol.34, no.3, pp.345-353, 1998.

[4] J.-X. Xu and Y. Tan. A composite energy function-based learning control approach for nonlinear systems with time-varying parametric uncertainties, IEEE Transactions on Auto-matic Control, vol. 47, no. 11, pp. 1940-1945, 2002.

[5] Tayebi. Adaptive iterative learning control for robot manip-ulators, Automatica, vol.40, no.7, pp.1195-1203, 2004.

[6] R. H. Chi, Z. S. Hou, and J. X. Xu. AdaptiveILCforaclass of discrete-time systems with iteration-varying trajectory and random initial condition, Automatica, vol.44, no.8, pp.2207-2213, 2008.

[7] C.-J. Chien. A combined adaptive law for fuzzy iterative learning control of nonlinear systems with varying control tasks, IEEE Transactions on Fuzzy Systems, vol.16, no.1, pp.40-51, 2008.

[8] Y.-C. Wang and C.-J. Chien. Decentralized adaptive fuzzy neural iterative learning control for nonaine nonlinear inter-connected systems, Asian Journal of Control, vol.13, no.1, pp.94-106, 2011.

[9] J.-X. Xu, D. Huang, V. Venkataramanan, and T. C. T. Huynh. Extreme precise motion tracking of piezoelectric positioning stage using sampled-data iterative learning control, IEEE Transactions on Control Systems Technology, vol.21, no.4, pp.1432-1439, 2013.

[10] Q. Lv, Y.-C. Fang, and X. Ren. Iterative learning control for accelerated inhibition effect of initial state random error, Acta Automatica Sinica, vol.40, no.7, pp.1295-1302, 2014.

[11] J.-M. Wei, Y.-A. Hu, and M.-M. Sun. Observer-based adaptive iterative learning control for a class of nonlinear time delay systems with input saturation, Mathematical Problems in Engineering, vol. 2015, Article ID 645161, 19 pages, 2015.

[12] J. Li and J. Li, Iterative learning control approach for a kind of heterogeneous multi-agent systems with distributed initial state learning, Applied Mathematics and Computation,vol.265, pp.1044-1057, 2015.

[13] L. Yan and J. Wei. Fractional order nonlinear systems with delay in iterative learning control, Applied Mathematics and Computation, vol.257, pp.546-552, 2015.

[14] H. Cai, Y. Huang, J. Du, T. Tang, D. Zuo, and J. Li. Iterative learning control with extended state observer for telescope sys-tem, Mathematical Problems in Engineering, vol.2015, Article ID 701510, 8 pages, 2015.

[15] Zhang Qunli. The effect of initial state error for nonlinear systems with delay via iterative learning control, Advances in Mathematical Physics, Volume 2016, Article ID 461950, 6pages, 2016.

[16] D. D. Bainov and P. S. Simeonov. Stability with respect to part of the variables in systems with impulse effect, Journal of Mathematical Analysis and Applications, 124(2), 547-560, 1987.

[17] G.K. Kulev and D. D. Bainov. Second method of Lyapunov and comparison principle for systems with impulse effect, Journal of Computation and Applied Mathematics, 23(3), 305-321, 1988.

[18] D. D. Bainov and P. S. Simeonov. Systems with Impulsive Effect: Stability Theory and Applied, Ellis Horwood, Chichester, UK, 1989.

[19] D. D. Bainov and P. S. Simeonov. Theory of Impulsive Differential Equations: Periodic Solution and Applications, Longman, Harlow, UK, 1993.
[20] I. M. Stamova. Vector Lyapunov functions for practical stability of nonlinear impulsive functional differential equations, Journal of Mathematical Analysis and Applications, 325(1), 612-623, 2007.

[21] S. Xinyu, G. Hongjian, and S. Xiangyun. Theory and Application of Impulsive Differential Equations, Science Press, Beijing, China, 2011.

[22] Q. Zhang, A class of vector Lyapunov functions for stability analysis of nonlinear impulsive differential systems, Mathematical Problems in Engineering, vol. 2014, Article ID 649012, 2014, 9 pages.

[23] M. X. Sun and B. J. Huang. Iterative learning control, National Defence Industry Press, Beijing, China, 1999. 